\title{
Same ion channel populations and different excitabilities: beyond the conductance-based model
}

\author{
Marco Arieli Herrera Valdez ${ }^{1,2,3}$ \\ From Twenty First Annual Computational Neuroscience Meeting: CNS*2012 \\ Decatur, GA, USA. 21-26 July 2012
}

Multicellular organisms rely on electrical signaling to communicate messages within and between different tissues. Channel-mediated ionic transport is typically modeled with conductance-based formulations that assume currents are the result of ionic electrical drift, without taking diffusion into consideration [1]. In contrast, formulations of current that assume ionic flux depends on electrical drift and diffusion are not as widely used in the literature [3-5]. These representations are more realistic and display experimentally observable phenomena that conductance-based models cannot reproduce (e.g. rectification). The two formulations are, however, mathematically related because conductance-based currents are linear approximations of drift-diffusion currents. Importantly, conductance-based models of membrane potential are not first order approximations of drift-diffusion models.

The two approaches predict qualitatively and quantitatively different behaviors in the dynamics of membrane potential. For instance, two neuronal membrane models with identical populations of ion channels, one written with conductance-based currents, the other with driftdiffusion currents, undergo transitions into and out of repetitive oscillations through different mechanisms and for different levels of stimulation. These differences in excitability are observed across different levels of ion channel expression in response to excitatory synaptic input. The electrophysiological profiles of drift-diffusion and conductance-based models having identical ion channel populations are different. As a consequence, the inputoutput and computational properties of networks constructed with conductance-based and electrodiffusion models should be different as well. The drift-diffusion formulation is proposed as a theoretical improvement over conductance-based models that may lead to more accurate predictions and interpretations of experimental data at the single cell and network levels.

\section{Acknowledgements}

The authors thank faculty and staff of the Institute of Interdisciplinary Research for their support. This work was supported in part by the Building Research Infrastructure and Capacity (BRIC) program at UPR-Cayey (P20 MD006144) through the National Institute of Minority Health and Health Disparities.

\section{Author details}

'Department of Mathematics, University of Arizona, Tucson, AZ, 85719, USA. 2Department of Mathematics and Physics, University of Puerto Rico at Cayey, Cayey, PR, 00736, USA. ${ }^{3}$ Institute of Interdisciplinary Research, University of Puerto Rico at Cayey, Cayey, PR, 00736, USA.

Published: 16 July 2012

\section{References}

1. Hodgkin AL, Huxley AF: A quantitative description of membrane current and its application to conduction and excitation in nerve. Journal of Physiology 1952, 117:500-544.

2. Goldman DE: Potential, impedance, and rectication in membranes. Journal of general Physiology 1943, 27:37.

3. Endresen LP, Hall K, Hoye JS, Myrheim J: A theory for the membrane potential of living cells. European Journal of Biophysics 2000, 29:90-103.

4. Herrera-Valdez MA: Geometry of reduced biophysical models of membrane potential. Dissertation, University of Arizona 2012.

5. Herrera-Valdez MA: Membranes with the same ion channel populations but different excitabilities. In review 2012.

doi:10.1186/1471-2202-13-S1-P120

Cite this article as: Herrera Valdez: Same ion channel populations and different excitabilities: beyond the conductance-based model. BMC Neuroscience 2012 13(Suppl 1):P120. 